\title{
Clinical sequencing: is WGS the better WES?
}

\author{
Janine Meienberg ${ }^{1} \cdot$ Rémy Bruggmann $^{2} \cdot \operatorname{Konrad~Oexle~}^{1} \cdot$ Gabor Matyas $^{1,3}$
}

Received: 1 September 2015 / Accepted: 25 December 2015 / Published online: 7 January 2016

(C) The Author(s) 2016. This article is published with open access at Springerlink.com

\begin{abstract}
Current clinical next-generation sequencing is done by using gene panels and exome analysis, both of which involve selective capturing of target regions. However, capturing has limitations in sufficiently covering coding exons, especially GC-rich regions. We compared whole exome sequencing (WES) with the most recent PCR-free whole genome sequencing (WGS), showing that only the latter is able to provide hitherto unprecedented complete coverage of the coding region of the genome. Thus, from a clinical/technical point of view, WGS is the better WES so that capturing is no longer necessary for the most comprehensive genomic testing of Mendelian disorders.
\end{abstract}

There is considerable discussion about the optimal application of next-generation sequencing (NGS) in the diagnosis of Mendelian disorders. Gene panels have been favored because of low sequencing costs, short turnaround time, and low rate of unspecific or incidental findings, while only

K. Oexle and G. Matyas have contributed equally to this work.

Electronic supplementary material The online version of this article (doi:10.1007/s00439-015-1631-9) contains supplementary material, which is available to authorized users.

Gabor Matyas

matyas@genetikzentrum.ch

1 Center for Cardiovascular Genetics and Gene Diagnostics, Foundation for People with Rare Diseases, 8952 Schlieren-Zurich, Switzerland

2 Interfaculty Bioinformatics Unit and Swiss Institute of Bioinformatics, University of Berne, 3012 Berne, Switzerland

3 Zurich Center for Integrative Human Physiology, University of Zurich, 8057 Zurich, Switzerland about $10 \%$ of the mutations detectable by whole exome sequencing (WES) were missed (Saudi Mendeliome Group 2015). In fact, gene panels related to the patients' phenotype can be viewed as an inexpensive and rapid first-tier test. If this test is negative, WES or whole genome sequencing (WGS) can be considered as the most comprehensive second-tier test.

In WGS, genome-wide read coverage may allow reliable detection of copy number variations (CNVs), which can contribute substantially to disease burden (Girirajan et al. 2011). The prices of WGS are tumbling, turnaround time including data analysis (e.g., using GENALICE MAP, www.genalice.com) can be reduced to few days, virtual gene panels can be selected in silico to avoid incidental findings, and diagnostic yield may be as high as $73 \%$, surmounting conventional phenotype-directed single-gene analyses by up to one order of magnitude (Soden et al. 2014; Miller et al. 2015; Willig et al. 2015). Thus, WGS has to be considered as an alternative to WES.

We recently showed that even current WES platforms have problems in sufficiently capturing the whole exome and suggested that WGS, which forgoes capturing, is less sensitive to GC content and more likely than WES to provide complete coverage of the entire coding region of the genome (Meienberg et al. 2015). Here, we provide new insights into WGS, showing that the recently introduced PCR-free WGS offers hitherto unprecedented complete coverage of the coding region of the genome and, hence, that WGS instead of WES should be considered as the most comprehensive second-tier test.

We compared optimal WES (using Agilent SureSelect v5 + UTR capturing; Meienberg et al. 2015) with WGS (using Illumina's TruSeq PCR-free WGS library preparation) in DNA samples of five females each. Sequencing was performed by vendors V2 (WES) and V4 (WGS) 

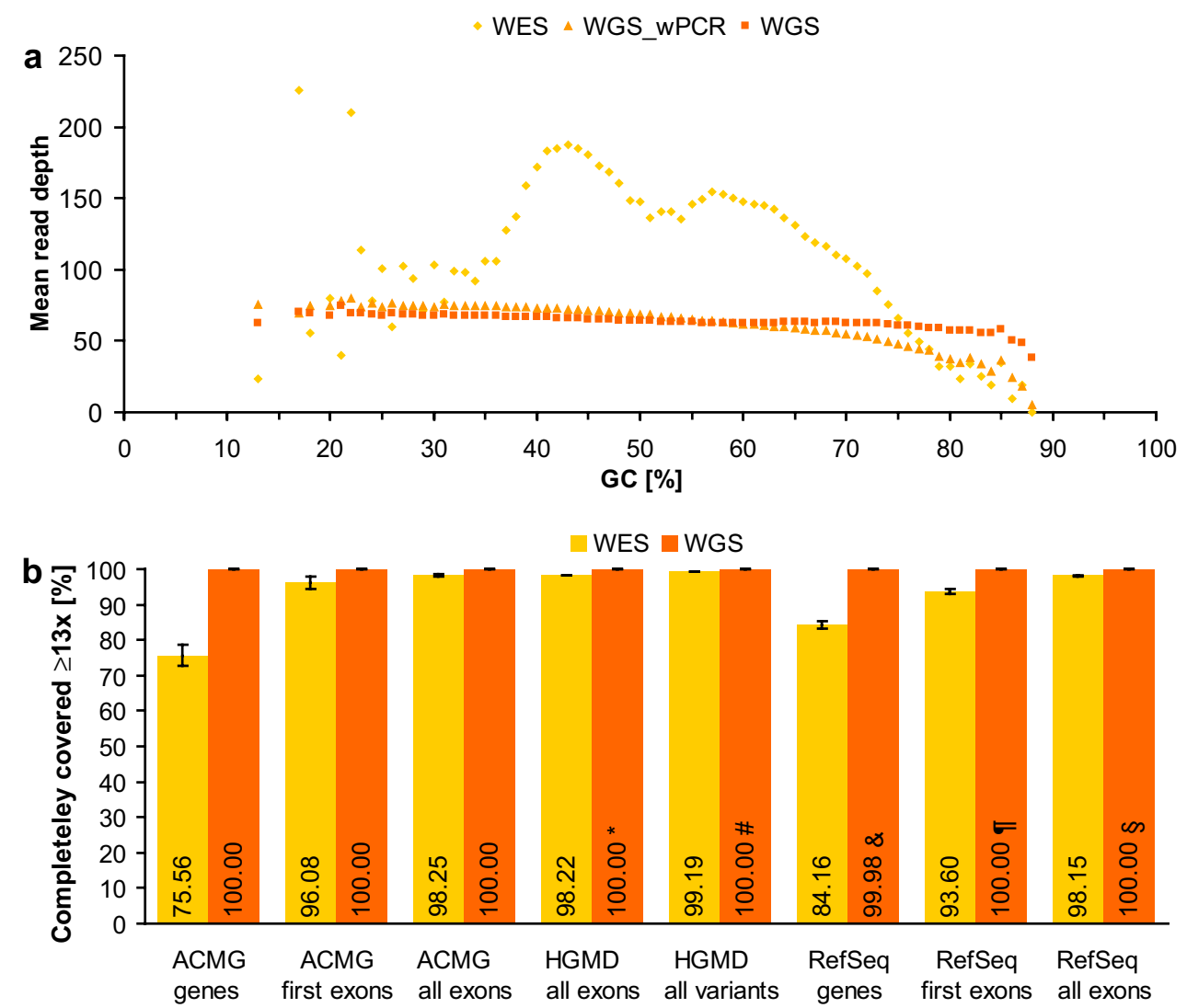

Fig. 1 Performance comparison of WES and WGS. a Mean read depth of RefSeq coding exons per GC content shown for WES as well as for WGS with (WGS_wPCR) and without (WGS) PCR as means of five samples each. b Percentage of completely covered (i.e. $\geq 13$ reads at each nucleotide position) genes, exons, and variants in WES and WGS without PCR as means of five samples each (error bars indicate $95 \%$ confidence intervals). In the case of genes recommended for reporting by the ACMG (ACMG genes, $n=54$ ) and of genes of the RefSeq database (RefSeq genes, $n=16,896$ ), the set of all coding exons (ACMG all exons, $n=1152$; RefSeq all exons,

on a HiSeq 2000 at $100 \times$ and a HiSeq $X$ Ten system at $60 \times$, respectively. To largely reduce systematic errors and alignment artifacts, we restricted our comparison to RefSeq coding sequences which were uniquely mappable to X-chromosomal or autosomal regions (Derrien et al. 2012), identical in hg19 and hg38 genome assemblies, and not overlapping with common CNVs listed in the Database of Genomic Variants (DGV, MacDonald et al. 2014). For further details see electronic supplementary material.

Our current data show that novel PCR-free WGS is much less sensitive to GC content and leads to a more uniform coverage than WES and non-PCR-free WGS (Fig. 1a, Supplementary Figs. S1-S3). Although the average depth of coverage was less than half $(65 \times$ in WGS versus $154 \times$ in WES, Supplementary Table S1), the number of RefSeq coding exons with complete $(100 \%)$ coverage at $\geq 13 \times$ was considerably larger in PCR-free WGS than in WES (100.00 $n=177,084)$ and the set of start-codon-containing exons (first exons) were examined. The set of RefSeq exons harboring at least one disease-causing mutation (DM) listed in HGMD (HGMD all exons, $n=22,303$ ) and the set of all coding and non-coding DMs (HGMD all variants, $n=106,819)$ were also analyzed. Note that $100.00 \%$ implies a deviation of at most $0.005 \%$ * *wo exons were partially covered with 12 reads; " one intronic mutation was covered with 12 reads; ${ }^{\&} 12$ genes were partially covered with $7-12$ reads; ${ }^{\mathbb{I}}$ three exons were partially covered with $10-12$ reads; ${ }^{\S} 12$ exons were partially covered with $7-12$ reads

vs. $98.15 \%$; Fig. 1b). The difference was more pronounced when the GC-rich first exons (59 vs. $51 \% \mathrm{GC}$ in all exons) were examined (100.00\% in PCR-free WGS vs. $93.60 \%$ in WES; Fig. 1b). In the case of genes recommended by the American College of Medical Genetics (ACMG) to be reported if mutated (Green et al. 2013), PCR-free WGS completely covered all uniquely mappable exons (100\% at $\geq 13 \times$ ) in all five samples of our study, whereas only $98.25 \%$ of the ACMG exons were completely covered by WES, leading to complete WES coverage of only $75.56 \%$ of the ACMG genes (Fig. 1b). A noticeable and clinically relevant difference in the performances of WES and WGS was also observed in the coverage of exons in which disease-causing mutations (DMs, including single nucleotide variants as well as small ( $\leq 20 \mathrm{bp})$ insertions, deletions, and indels) have been reported in HGMD (98.22 \% in WES vs. $100.00 \%$ in WGS; Fig. 1b). Accordingly, WES may 
fail to detect $0.42 \%(401 / 95,118)$ of the currently known exonic DMs detectable by WGS. Considering the identification of non-coding pathogenic variation as well (Spielmann and Klopocki 2013), WES may miss a total of $0.81 \%$ $(863 / 106,819)$ of the DMs currently listed in HGMD and potentially detectable by WGS $(99.19 \%$ in WES vs. all but one DM in WGS; Fig. 1b). Notably, the $13 \times$ cutoff presented here reveals the minimum number of reads at which WGS achieves $100.00 \%$ coverage in our samples. For the same WGS performance at the widely used $20 \times$ cutoff, sequencing at $>100 \times(65 * 20 / 13)$ is needed (while for WES more sequencing reads may not result in more complete coverage due to capture limitations, especially in GC-rich regions).

Furthermore, genome-wide uniformity of coverage makes WGS, rather than WES, suitable for CNV detection (Gilissen et al. 2014; Meienberg et al. 2015). In our samples, the coefficient of variation ( $\mathrm{cv}=\mathrm{SD} /$ mean) in coverage among the exons of an individual is on average about 4 times larger in WES than in PCR-free WGS (0.59 vs. 0.14). Admittedly, the relative lack of uniform coverage in WES does not appear to result from an increased noise level, since the inter-individual cv per exon is comparable in WES and WGS (0.08 vs. 0.09). In other words, the additional variability of WES coverage appears to be reproducible and, hence, can in principle be normalized in silico. However, such normalization algorithms are relatively complex, need to be calibrated for each enrichment protocol (Szatkiewicz et al. 2013), and allow only the detection of CNVs affecting the enriched genomic region. Moreover, gapless WGS also offers the detection of structural variants (SVs) based on paired and split reads, enabling the detection of (copy neutral) SVs at base-pair resolution (Escaramis et al. 2015). Thus, in our opinion, WGS will likely replace array techniques in $\mathrm{CNV}$ detection whereas WES might not.

WGS is available worldwide in laboratories that have high-throughput sequencing capacities of at least $60 \times 3 * 10^{9} \mathrm{bp}$ as well as appropriate hard- and software resources to handle and interpret large WGS files. One may argue that WGS is more expensive than NGS with selective capturing of targets. Indeed, genetic mosaics and somatic cancer gene panels require several 100 -fold sequencing depths to detect low-frequency non-reference variants, so that WGS would currently be too expensive for these applications. Otherwise, however, sequencing costs decline steadily and data interpretation efforts can be curtailed by in silico selection of relevant WGS parts. Considering that these parts are subject to change, selective capturing will require resequencing of unsolved cases, while with WGS only the reanalysis of existing data will be necessary. In addition, one may argue that WGS implies incidental findings of mutations not related to the patient's present disease and findings of variants with uncertain or incomplete effect. Again, overload with such findings can be prevented by reducing the
WGS data to virtual gene panels of interest. Thus, we and others (Belkadi et al. 2015; Lelieveld et al. 2015) believe that WGS is more powerful than WES in detecting exome variants so that future NGS diagnostics of Mendelian disorders will not involve capturing techniques anymore. In addition to previous studies, our present data show that PCR-free WGS provides an even more uniform and complete coverage of the exome than WGS with PCR during library preparation.

In conclusion, the performance of WES is sensitive to sequence (GC) content as well as capturing design and enrichment. Hence, WES does not entirely serve its purpose, whereas novel PCR-free WGS provides hitherto unprecedented complete coverage of the exome and other clinically relevant genomic sequences. The advantage of WGS therefore does not only include the identification of non-coding pathogenic variation, but, in view of its more complete exomic coverage as presented here, it is simply the better WES. As such, PCR-free WGS has to be considered as the most comprehensive second-tier genomic test. With sequencing costs further declining and by using appropriate virtual panels, WGS even has the potential to entirely replace WES and other techniques that involve selective capturing of target sequences.

Acknowledgements We thank the two sequencing vendors (V2 and V4) involved in this study for performing WES and WGS. This study was supported by the Bangerter-Rhyner-Stiftung, COFRA Foundation, Ebnet-Stiftung, Gebauer Stiftung, Hirzel-Callegari Stiftung, Spendenstiftung Bank Vontobel, and Stiftung FERNE HORIZONTE.

Conflict of interests The authors declare that there is no conflict of interests.

Open Access This article is distributed under the terms of the Creative Commons Attribution 4.0 International License (http://creativecommons.org/licenses/by/4.0/), which permits unrestricted use, distribution, and reproduction in any medium, provided you give appropriate credit to the original author(s) and the source, provide a link to the Creative Commons license, and indicate if changes were made.

\section{References}

Belkadi A, Bolze A, Itan Y et al (2015) Whole-genome sequencing is more powerful than whole-exome sequencing for detecting exome variants. Proc Natl Acad Sci USA 112:5473-5478

Derrien T, Estelle J, Marco Sola S et al (2012) Fast computation and applications of genome mappability. PLoS One 7:e30377

Escaramis G, Docampo E, Rabionet R (2015) A decade of structural variants: description, history and methods to detect structural variation. Brief Funct Genomics 14:305-314

Gilissen C, Hehir-Kwa JY, Thung DT et al (2014) Genome sequencing identifies major causes of severe intellectual disability. Nature 511:344-347

Girirajan S, Brkanac Z, Coe BP et al (2011) Relative burden of large $\mathrm{CNVs}$ on a range of neurodevelopmental phenotypes. PLoS Genet 7:e1002334 
Green RC, Berg JS, Grody WW et al (2013) ACMG recommendations for reporting of incidental findings in clinical exome and genome sequencing. Genet Med 15:565-574

Lelieveld SH, Spielmann M, Mundlos S, Veltman JA, Gilissen C (2015) Comparison of exome and genome sequencing technologies for the complete capture of protein-coding regions. Hum Mutat 36:815-822

MacDonald JR, Ziman R, Yuen RK, Feuk L, Scherer SW (2014) The Database of Genomic Variants: a curated collection of structural variation in the human genome. Nucleic Acids Res 42:D986-D992

Meienberg J, Zerjavic K, Keller I et al (2015) New insights into the performance of human whole-exome capture platforms. Nucleic Acids Res 43:e76

Miller NA, Farrow EG, Gibson M et al (2015) A 26-hour system of highly sensitive whole genome sequencing for emergency management of genetic diseases. Genome Med 7:100
Saudi Mendeliome Group (2015) Comprehensive gene panels provide advantages over clinical exome sequencing for Mendelian diseases. Genome Biol 16:134

Soden SE, Saunders CJ, Willig LK et al (2014) Effectiveness of exome and genome sequencing guided by acuity of illness for diagnosis of neurodevelopmental disorders. Sci Transl Med 6:265ra168

Spielmann M, Klopocki E (2013) CNVs of noncoding cis-regulatory elements in human disease. Curr Opin Genet Dev 23:249-256

Szatkiewicz JP, Wang W, Sullivan PF, Sun W (2013) Improving detection of copy-number variation by simultaneous bias correction and read-depth segmentation. Nucleic Acids Res 41:1519-1532

Willig LK, Petrikin JE, Smith LD et al (2015) Whole-genome sequencing for identification of Mendelian disorders in critically ill infants: a retrospective analysis of diagnostic and clinical findings. Lancet Respir Med 3:377-387 